\title{
Partial Fermionization: Spectral Universality in 1D Repulsive Bose Gases
}

\author{
Quirin Hummel, Juan Diego Urbina, and Klaus Richter \\ Institut für Theoretische Physik, Universität Regensburg, D-93040 Regensburg, Germany
}

(Received 4 December 2018; revised manuscript received 26 March 2019; published 17 June 2019)

\begin{abstract}
Because of the vast growth of the many-body level density with excitation energy, its smoothed form is of central relevance for spectral and thermodynamic properties of interacting quantum systems. We compute the cumulative of this level density for confined one-dimensional continuous systems with repulsive short-range interactions. We show that the crossover from an ideal Bose gas to the strongly correlated, fermionized gas, i.e., partial fermionization, exhibits universal behavior: Systems with very few and up to many particles share the same underlying spectral features. In our derivation we supplement quantum cluster expansions with short-time dynamical information. Our nonperturbative analytical results are in excellent agreement with numerics for systems of experimental relevance in cold atom physics, such as interacting bosons on a ring (Lieb-Liniger model) or subject to harmonic confinement. Our method provides predictions for excitation spectra that enable access to finite-temperature thermodynamics in large parameter ranges.
\end{abstract}

DOI: 10.1103/PhysRevLett.122.240601

The huge progress in cold atom physics has enabled precision experiments which allow us to confine, control, and study ensembles of atoms with particle numbers ranging from very few [1-5] to nearly macroscopically many [6-9]. The high control over parameters, trapping to low dimensions, and tunability of interactions has lead to a synergetic understanding of highly correlated many-body (MB) systems [10], in many cases based on theories of onedimensional integrable models $[11,12]$ and correspondingly tailored experiments $[13,14]$. However, in situations deviating from integrability (see, e.g., Refs. [15-19]) the theoretical treatment of systems with an intermediate number $N$ of interacting identical particles is particularly hard, especially when the observed spectral, thermodynamic, or dynamical properties involve highly excited multiparticle states.

The conceptual challenges are numerous: First, systems with fixed $N$ require a canonical treatment, in particular when approaching the few-body regime, where grand canonical approaches often fail [20]. Second, due to strong inter-particle correlations that can experimentally be pushed up to the limit of fermionization in Bose gases $[3,7,8,21,22]$, and especially for small $N$, mean-field approaches or more generally $1 / N$ expansions get problematic. Elaborate $\mathrm{MB}$ techniques allow for calculating ground and low excited states of such interacting multiparticle systems with high precision (see, e.g., Refs. $[23,24])$. However, these methods reach their limits when increasing $N$ or the degree of excitation since this implies vastly growing Hilbert space dimensions.

This goes along with a close to exponential increase of the MB density of states (DOS) with excitation energy for continuous $N$-particle systems, even in the $1 \mathrm{D}$ case. The universal Bethe law [25,26] and variants [27,28] for sufficiently low-lying excitations in large- $N$ fermionic systems represent a famous example in nuclear physics. There, the effect of (residual) interactions is merely a broadening of the otherwise highly degenerate noninteracting MB spectrum [29,30], while for small to intermediate $N$ interactions have nontrivial effects and the Bethe law generally fails $[31,32]$.

Nonetheless, the spacing between MB levels as well as the associated fluctuations tend to zero such that individual highly excited MB levels are usually no longer resolvable. Hence the (locally) energy-averaged, smooth MB DOS $\rho^{(N)}(E)$ gains particular relevance [33]. In particular it plays the central role for computing thermodynamic equilibrium properties at finite temperature. Beyond that, $\rho^{(N)}(E)$ is a key ingredient to nonequilibrium quantum work statistics that has drawn much attention lately [36-39], not least due to a recently revealed connection to information scrambling [40].

This calls for developing genuinely interacting MB techniques specifically devised to directly compute the smooth DOS, thereby circumventing the intricate [41-44] or simply impossible calculation of individual (highly) excited MB levels which requires additional information that is afterwards smoothed out anyway.

Similar to the single particle case [45-48], a smooth MB DOS corresponds to and requires dynamical information from $\mathrm{MB}$ quantum propagation on finite time scales only. Invoking such short-time information in a quantum cluster expansion (QCE) [49-51] implies, as we will show, that interaction effects in the smooth DOS arise 


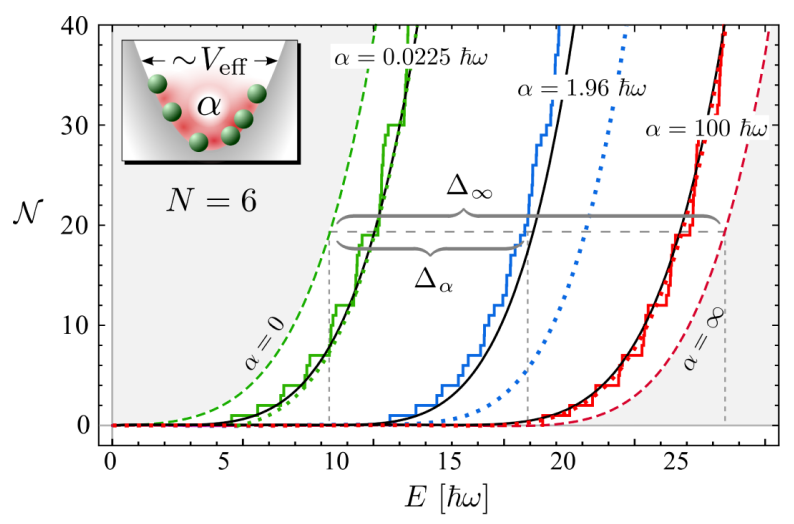

FIG. 1. Many-body level counting function for six interacting bosons in a harmonic trap (spacing $\hbar \omega$ ) for different contact interaction strengths $\alpha$. Numerically exact results for $\mathcal{N}(E)$ (staircases) exhibit characteristic shifts $\Delta_{\alpha}$ in $E$ towards the limit $\alpha \rightarrow \infty$ of full fermionization. These shifts carry universal features and are quantitatively explained by our theory (solid lines) based on Eqs. (8) and (11). Dotted lines denote analytical QCE-based approximations [Eq. (5)] invoking the limiting cases of weak and strong $\alpha$, see main text.

nonperturbatively from universal cluster kernels dressed with terms depending on the confinement potential, required to be a homogeneous function. Specifically, we consider not directly the MB DOS $\rho^{(N)}(E)$ but the (smooth) MB level counting function $\mathcal{N}(E)=\int_{0}^{E} d E^{\prime} \rho^{(N)}\left(E^{\prime}\right)$, depicted in Fig. 1 for a harmonically trapped Bose gas. $\mathcal{N}(E)$ exhibits interaction-dependent characteristic horizontal shifts $\Delta_{\alpha}$ indicating what we call partial fermionization. We will analytically show that these shifts, and thereby $\mathcal{N}$ and $\rho^{(N)}$, follow with high accuracy $N$-independent universal laws, i.e., broad classes of interacting bosonic systems ranging from very few to many particles possess equal spectral features. Remarkably, these robust features are reminiscent of the spectral shifts in the famous solvable CalogeroSutherland models $[52,53]$ which admit an interpretation in terms of fractional exclusion statistics [54-56].

We first outline the main steps of our QCE for the canonical partition function providing the basis for our further (asymptotic) analysis to derive our main result, a universal law for partial fermionization.

Canonical partition function.-The MB DOS $\rho_{ \pm}^{(N)}(E)$ of a system of $N$ identical quantum particles (" \pm " denoting bosons and fermions) is related to the canonical partition function $Z_{ \pm}^{(N)}(\beta)$ through the inverse Laplace transform $\rho_{ \pm}^{(N)}(E)=\mathcal{L}_{\beta}^{-1}\left[Z_{ \pm}^{(N)}(\beta)\right](E)$ with $\beta=1 /\left(k_{\mathrm{B}} T\right)$. Furthermore, $Z_{ \pm}^{(N)}(\beta)=\operatorname{Tr}_{ \pm} K^{(N)}(t=-i \hbar \beta)$ is the trace over the propagator $K^{(N)}$ for $N$ distinguishable particles in the properly (anti-)symmetrized basis.

For $N$ noninteracting particles of mass $m$, each with coordinates $\mathbf{q}$, confined by a homogeneous potential $U(\mathbf{q})=w^{\mu} U(\mathbf{q} / w)$, it can be expressed in closed form [31,57],

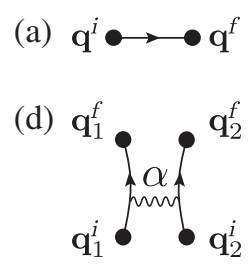

(b)

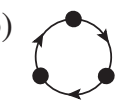

(e)

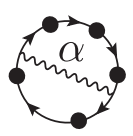

(c)

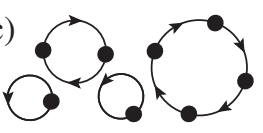

(f)

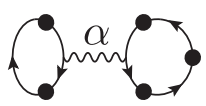

FIG. 2. Leading-order contributions to the quantum cluster expansion. (a) SP propagator $K_{0}^{(1)}\left(\mathbf{q}^{f}, \mathbf{q}^{i} ; t\right)$; (b) contribution $\mathcal{A}_{n}$ from a single cycle (here $n=3$ ); (c) specific clustering yielding $\mathcal{A}_{\mathfrak{N}}$ (here $\mathfrak{N}=\{1,1,2,4\}, N=8$ ); (d) interacting part $\Delta K_{\alpha}^{(2)}\left(\left(\mathbf{q}_{1}^{f}, \mathbf{q}_{2}^{f}\right),\left(\mathbf{q}_{1}^{i}, \mathbf{q}_{2}^{i}\right) ; t\right)$ of the two-body propagator; (e),(f) examples for intra- and inter-cycle contributions $\mathcal{A}_{n_{1}, n_{2}}^{\text {intra }}$ and $\mathcal{A}_{n_{1}, n_{2}}^{\text {inter }}$ with $n_{1}=3, n_{2}=2$. A diagram is evaluated as the product of all SP and interacting two-body components $K_{0}^{(1)}$ and $\Delta K_{\alpha}^{(2)}$ after spatial integration over all (internal) points.

$$
Z_{0, \pm}^{(N)}(\beta)=\sum_{l=1}^{N} z_{ \pm, l}^{(N, d)}\left(\frac{V_{\mathrm{eff}}}{\lambda_{T}^{d}}\right)^{l}
$$

with universal constants $z_{ \pm, l}^{(N, d)}$, physical dimension $D$, and effective dimension $d=D[1+(2 / \mu)]$. Setting $\hbar^{2} /(2 m)=1$, the thermal wavelength is $\lambda_{T}=\sqrt{4 \pi \beta}$ and the effective volume is $V_{\text {eff }}=(4 \pi)^{D / \mu} \int d^{D} q \exp [-U(\mathbf{q})]$. The case without external potential is included as $\mu \rightarrow \infty$, then $d=D$ and $V_{\text {eff }}$ equals the physical volume $V_{D}$.

Quantum cluster expansion.-The noninteracting part $K_{0}^{(N)}$ of the propagator factorizes into single-particle (SP) propagators, see Fig. 2(a). A contribution to $Z_{0, \pm}^{(N)}$ corresponding to a permutation $P$ is a product of cluster terms, resembling the decomposition of $P$ into cycles [62]. Using the semigroup property of the SP propagator and identifying $\mathbf{q}_{n+1} \equiv \mathbf{q}_{1}=\mathbf{q}$, each cycle involving a subset of $n$ particles [see Fig. 2(b)] yields the amplitude $\mathcal{A}_{n}(t)=$ $\int d^{D} q K_{0}^{(1)}(\mathbf{q}, \mathbf{q} ; n t)$. In line with our major assumption of short-time propagation we can use [31] $K_{0}^{(1)}(\mathbf{q}, \mathbf{q} ; t) \simeq$ $\exp [-(i / \hbar) U(\mathbf{q}) t] K_{\text {free }}^{(1)}(\mathbf{q}, \mathbf{q} ; t)$ where $K_{\text {free }}$ stands for unconfined propagation. The full contribution to $Z_{0, \pm}^{(N)}$ of a permutation is then $\mathcal{A}_{\mathfrak{N}}(-i \hbar \beta)=\prod_{n \in \mathfrak{N}} \mathcal{A}_{n}(-i \hbar \beta)$, in terms of the multiset $\mathfrak{N}=\left\{n_{1}, n_{2}, \ldots, n_{|\mathfrak{N}|}\right\}$ of cycle lengths, see Fig. 2(c). Further evaluation of these amplitudes eventually yields the explicit result Eq. (1) [31,57].

The implementation of interaction effects begins with a cluster expansion [49-51] of $K^{(N)}$ to first order in the interaction by decomposing the full two-body propagator $K^{(2)}=K_{0}^{(2)}+\Delta K_{\alpha}^{(2)}$ into $K_{0}^{(2)}$ and nonperturbative interaction contributions $\Delta K_{\alpha}^{(2)}$ where $\alpha$ is an energy associated with the coupling strength [63]. To calculate interaction effects we choose all pairs $\{k, l\}$ of particles and replace $K_{0}^{(1)}\left(\mathbf{q}_{P(k)}, \mathbf{q}_{k} ; t\right) K_{0}^{(1)}\left(\mathbf{q}_{P(l)}, \mathbf{q}_{l} ; t\right)$ in $\mathcal{A}_{\mathfrak{N}}$ by the interaction term $\Delta K_{\alpha}^{(2)}\left(\left(\mathbf{q}_{P(k)}, \mathbf{q}_{P(l)}\right),\left(\mathbf{q}_{k}, \mathbf{q}_{l}\right) ; t\right)$, see Fig. 2(d). The 
interaction can link two particles involved in either the same [see Fig. 2(e)] or in two different cycles [see Fig. 2(f)] of $P$, referred to as intra- and inter-cycle contributions $\mathcal{A}_{n_{1}, n_{2}}^{\text {intrater }}$ where $n_{1}, n_{2}$ denotes the distribution of the $n=$ $n_{1}+n_{2}$ particles. Evaluation of the diagram classes in Figs. 2(e) and 2(f) yields

$$
Z_{\alpha, \pm}^{(N)}=Z_{0, \pm}^{(N)}+\sum_{n=2}^{N}( \pm 1)^{n} Z_{0, \pm}^{(N-n)} \sum_{n_{1}=1}^{n-1} \mathcal{A}_{n_{1}, n-n_{1}}^{ \pm}
$$

with amplitudes of the form

$$
\mathcal{A}_{n_{1}, n_{2}}^{ \pm}=\frac{1}{2}\left[\mathcal{A}_{n_{1}, n_{2}}^{\mathrm{inter}} \pm \mathcal{A}_{n_{1}, n_{2}}^{\mathrm{intra}}\right]=\frac{V_{\mathrm{eff}}}{\lambda_{T}^{d} n^{d / 2}} a_{n_{1}, n_{2}}^{ \pm}(\beta \alpha),
$$

defining the interaction kernels $a_{n_{1}, n_{2}}^{ \pm}(\beta \alpha)$, see below.

The philosophy behind cluster expansions implies that the form Eq. (3) of amplitudes is generic for arbitrary shortrange interactions [64]: The main contribution to the $n$-fold integrals involved stems from the region where all $n$ particles are close to each other, allowing us to extend all integrals over relative coordinates to infinity. Hence the $a_{n_{1}, n_{2}}^{ \pm}(\beta \alpha)$ do not depend on the external potential, a key feature of our approach that also generalizes to higher order, i.e., clusters involving $n$-body corrections. Instead, only the center of mass is subject to $U(\mathbf{q})$, thus yielding the effective system size $V_{\text {eff }}$ as a prefactor in Eq. (3). In view of Eqs. (1)-(3) this yields the entire QCE partition function

$$
Z_{\alpha, \pm}^{(N)}(\beta)=\sum_{l=1}^{N}\left[z_{ \pm, l}^{(N, d)}+\Delta z_{ \pm, l}^{(N, d)}(\beta \alpha)\right]\left(\frac{V_{\text {eff }}}{\lambda_{T}^{d}}\right)^{l}
$$

with interaction-related terms $\Delta z_{ \pm, l}^{(N, d)}(\beta \alpha)$ given by the kernels, i.e., to first order, $a_{n_{1}, n_{2}}^{ \pm}(\beta \alpha)$ [57].

Correspondingly, the general QCE expression for the central quantity $\mathcal{N}_{\alpha}(E)=\int_{0}^{E} d E^{\prime} \rho^{(N)}\left(E^{\prime}\right)$ is [57,64], to arbitrary order,

$\mathcal{N}_{\alpha}(E)=\sum_{l=1}^{N}\left[\frac{z_{ \pm, l}^{(N, d)}}{\Gamma\left(\frac{l d}{2}+1\right)}+g_{ \pm, l}^{(N, d)}\left(\frac{E}{\alpha}\right)\right] V_{\mathrm{eff}}^{l}\left(\frac{E}{4 \pi}\right)^{l d / 2}$.

It features the same polynomial structure in $V_{\mathrm{eff}} E^{d / 2}$ as its noninteracting counterpart, while the $g_{ \pm, l}^{(N, d)}$ add a functional dependence on $E / \alpha$ to the coefficients given by the interaction kernels, i.e., to first order, $a_{n_{1}, n_{2}}^{ \pm}(\beta \alpha)$.

Contact interaction.-For explicit calculations and motivated by the central importance for quasi-1D cold atom systems $[10,65,66]$ we consider Hamiltonians

$$
\hat{H}=\sum_{i=1}^{N}\left(-\frac{\partial^{2}}{\partial q_{i}^{2}}+U\left(q_{i}\right)\right)+\sqrt{8 \alpha} \sum_{i<j} \delta\left(q_{i}-q_{j}\right)
$$

of $N$ interacting bosons with coordinates $q_{i}$ in 1D. One obtains [57] explicit analytical expressions for the kernels $a_{n_{1}, n_{2}}^{+}(\beta \alpha)$ in Eq. (3). Closed explicit expressions for the $g_{ \pm, l}^{(N, d)}(E / \alpha)$ in Eq. (5) follow for the prominent 1D cases of $U(q)=0(d=1)$, harmonic confinement $(d=2)$, and linear potential wells $(d=3)$ [57].

Before addressing representative cases we note that the QCE [Eqs. (4) and (5)], evaluated to first order, although devised for weak interaction, can also be applied to the complementary regime of strong coupling [57] by means of fermionization $[67,68]$ due to an exact duality [69] of strongly coupled bosons and weakly coupled spinless fermions.

Harmonic confinement.-We first consider $U\left(q_{i}\right)=$ $(\hbar \omega)^{2} q_{i}^{2} / 4$, for which $V_{\text {eff }}=4 \pi /(\hbar \omega)$, and compare in Fig. 1 analytical QCE results (dotted lines) for $\mathcal{N}_{\alpha}(E)$ with extensive numerical calculations (staircases) based on exact diagonalization and hence restricted to roughly the first 40 excited MB levels for $N=6$. The first-order QCE, implemented as weak- and dual strong-coupling expansions, indeed is valid in the respective regimes. However, for intermediate couplings (here $\alpha \simeq 2 \hbar \omega$ ) it degrades. Moreover, such deviations grow with increasing $N$ calling for an improved method that adequately treats intermediate couplings.

Partial fermionization.-Interactions predominantly cause characteristic shifts of $\mathcal{N}_{\alpha}(E)$ towards larger energies (as visible in Fig. 1). Presuming knowledge of the noninteracting spectra, the shifts $\Delta_{\alpha}$ of individual levels contain all information about the interacting spectra. We adopt this reformulation of the problem to develop a method that directly addresses these shifts on average. Our approach further enables asymptotic considerations that strongly simplify the MB problem and highlight the universality behind partial fermionization.

For the interaction-induced energy shift at fixed $\mathcal{N}$,

$$
\Delta_{\alpha} \equiv\left\langle E^{(n)}(\alpha)-E^{(n)}(0)\right\rangle_{n} \equiv\left\langle E^{(n)}(\alpha)\right\rangle_{n}-E_{0},
$$

averaged over a bunch of individual MB levels $E^{(n)}$ we propose, in a first-order implementation, the ansatz

$$
\Delta_{\alpha} \approx \chi^{(N, d)}(E / \alpha) \Delta_{\infty}^{(N, d)}\left(E_{0}, V_{\mathrm{eff}}\right),
$$

where $E=E_{0}+\Delta_{\alpha}$ is the shifted energy, separating the $V_{\text {eff }}$ dependence from an $\alpha$-dependent function $\chi^{(N, d)}$, in view of the notable structure of $\mathcal{N}_{\alpha}$ within QCE [Eq. (5)] and corroborated by a general consistency argument $[57,64] . \Delta_{\infty}$ denotes the full "horizontal" shift (see Fig. 1) between fermionized and noninteracting bosonic levels for fixed $\mathcal{N} \equiv\langle n\rangle_{n}=\mathcal{N}_{0}\left(E_{0}\right)$. We find [57]

$$
\Delta_{\infty}^{(N, d)} \approx \text { const } \times V_{\mathrm{eff}}^{-2 / d} \mathcal{N}^{(2 / d-1) / N} .
$$


The $\alpha$-dependent factor $\chi \in[0,1]$ in Eq. (8) continuously interpolates between the free Bose gas $\chi \rightarrow 0$ and the fully fermionized gas $\chi \rightarrow 1$, quantifying partial fermionization. Most notably, the central function $\chi^{(N, d)}(E / \alpha)$ in Eq. (8) is uniquely obtained from QCE [Eq. (5)] by matching

$$
\mathcal{N}_{\alpha}(E)=\mathcal{N}=\mathcal{N}_{0}\left(E_{0}\right)=\mathcal{N}_{0}\left(E-\Delta_{\alpha}\right)
$$

in the regime $E^{d / 2} V_{\text {eff }} \gg 1$ of weak quantum degeneracy, where the first-order QCE becomes increasingly accurate. For the lhs of Eq. (10) we apply QCE [Eq. (5)], while for the rhs we use the result Eq. (1) for $\alpha=0$, and implement the shift $\Delta_{\alpha}$, Eq. (8), as an expansion around $E$ in the small parameter $\Delta_{\infty}^{(N, d)} / E=\mathcal{O}\left(E^{-d / 2} V_{\text {eff }}^{-1}\right)$. Matching the next-toleading order $\mathcal{O}\left(V_{\mathrm{eff}}^{N-1}\right)$ in Eq. (10) fixes $\chi^{(N, d)}(E / \alpha) \propto$ $-g_{+, N-1}^{(N, d)}(E / \alpha)$ [57], which, remarkably, is fully determined by two-body clusters for which the first-order QCE is exact.

A solution for $\mathcal{N}_{\alpha}(E)$ is achieved by determining the partial fermionization for a given initial noninteracting energy $E_{0}$, reducing the problem to finding, in view of Eq. (8), the root of

$$
x=\chi^{(N, d)}(E / \alpha)=\chi^{(N, d)}\left[\left(E_{0}+x \Delta_{\infty}\right) / \alpha\right] .
$$

This implicitly defines $x=\chi$ as a function of $E_{0}, \alpha$, and $N$. The method efficiently emulates the effect of higher-order clusters in terms of the smallest ones, giving excellent predictions (see solid curves in Fig. 1). While the presented lowest-order version involves only two-body clusters it can be pushed to second (and higher) order in a controlled way $[57,64]$ by extending the ansatz Eq. (8) to admit corrections to $\chi$ in powers of $\left(E_{0}^{\mathrm{sc}}\right)^{-d / 2}$ [see Eq. (13) below]. Those incorporate three-body (and larger) clusters that correct for multiparticle collision effects in the deeply quantum degenerate regime.

Asymptotics and universality.-An asymptotic analysis [57] of $\chi$ and $\Delta_{\infty}$ for large $N$ further reveals the existence of a specific finite limit, i.e., in first order,

$$
x \approx \lim _{N \rightarrow \infty} \chi^{(N, d)}(N \tilde{\epsilon})=1-e^{d /(2 \tilde{\epsilon})} \operatorname{erfc} \sqrt{d /(2 \tilde{\epsilon})},
$$

where $\tilde{\epsilon}=\left[E_{0}^{\mathrm{sc}}+x \Delta_{\infty}^{\mathrm{sc}}\left(E_{0}^{\mathrm{sc}}\right)\right] / \alpha^{\mathrm{sc}}$ and, with $n_{\mathrm{eff}}=N / V_{\mathrm{eff}}$, $E_{0}^{\mathrm{sc}}=\frac{E_{0}}{N} \mathcal{E}^{-1}, \quad \alpha^{\mathrm{sc}}=\alpha \mathcal{E}^{-1}, \quad \mathcal{E}=\frac{2 \pi \hbar^{2}}{m} n_{\mathrm{eff}}^{2 / d}$,

implying a universal law for the partial fermionization,

$$
\chi \stackrel{N \gg 1}{\approx} \chi\left(d, E_{0}^{\mathrm{sc}}, \alpha^{\mathrm{sc}}\right) .
$$

For systems with different $N \gg 1$ it predicts that $\chi$, and hence $\mathcal{N}_{\alpha}$, depend in the same peculiar way on $\alpha$ and the energy per particle $E_{0} / N$ of the corresponding noninteracting system, both appropriately scaled in terms of the energy unit $\mathcal{E}=\left(2 \pi \hbar^{2} / m\right) n_{\text {eff }}^{2 / d}$, establishing a key feature of the observed universality: It relates high excitations in large- $N$ systems to low-lying excitations in corresponding systems with smaller, but still considered large, $N$. Explicit approximants for $\chi\left(d, E_{0}^{\mathrm{sc}}, \alpha^{\mathrm{sc}}\right)$ can be found by iteration [57].

In Figs. 3(a) and 3(b) we compare these predictions with numerically obtained data based on MB levels of Eq. (6) for the paradigmatic Lieb-Liniger model [70-73] $\left[U\left(q_{i}\right)=0\right.$ on a ring with length $L$, i.e., $\left.n_{\text {eff }}=N / V_{\text {eff }}=N / L\right]$. We find that the universality is fulfilled with remarkable accuracy for the whole range of interactions and particle numbers, most notably even down to $N=2$. Moreover, spectral fluctuations, not included in our analytical approach, are strongly suppressed for growing $N$, implying approximate analytical predictability of individual excited MB energies for arbitrary parameters.

Inaccuracies at very low energies and couplings are cured by extending the energy shifting from first order (dashed line), based on two-body processes, to second order (solid line) involving three-cluster diagrams which, again, can be calculated analytically [64,74]. Our approach amounts to a description of the entire smooth spectrum in terms of only two- or three-body processes which nonperturbatively interpolates between $\alpha=0$ and $\alpha \rightarrow \infty$.

Figure 3(c) shows results for harmonic confinement, for which $\Delta_{\infty}^{\mathrm{sc}}=1 / 2$, representing a generic nonintegrable $N$-particle system, see also Fig. 1 . The full lines display the analytical solutions for finite $N=3,4,6,8$, converging to the universal large- $N$ limit Eq. (14). The universal prediction (inset) shows, besides fermionization $\chi \simeq 1$ (roof) and the perturbative regime $\chi \simeq 2 \sqrt{\alpha^{\mathrm{sc}} /\left(\pi E_{0}^{\mathrm{sc}}\right)}$ (right flank), a nonperturbative quantum regime for $E_{0}^{\mathrm{sc}} \ll\left(\alpha^{\mathrm{sc}}\right)^{1 / 3}, \alpha^{\mathrm{sc}} \ll 1$ where $\chi \simeq 2\left(\alpha^{\mathrm{sc}} / \pi\right)^{1 / 3}$ becomes independent of $E_{0}^{\text {sc }}$ [57]. This peculiarity connects our findings to the solvable Calogero-Sutherland models with $\sim 1 / r^{2}$ interaction $[52,53,75]$ where spectra are exact bosonic-to-fermionic interpolations that we identify as a specific realization of $\chi$ fulfilling universality, in this case level by level and constant in $E_{0}^{\mathrm{sc}}$. Here we find a generalization (including nonintegrable systems) where $\chi$ is allowed to vary over the (smoothed) spectrum in a way characteristic for the particular type of interaction. This illustrates that due to the generality of the QCE approach [Eq. (5)], the shifting procedure [Eqs. (8)-(11)], and the subsequent asymptotic analysis, universality [Eq. (14)] of $\chi$ is not restricted to contact interaction [Eq. (6)]. We stress that it also applies to higher-order implementations.

We close with a few remarks. (i) Our method provides predictions for regions of excitation spectra and particle numbers that are barely accessible via full numerical calculations. (ii) Universality [Eq. (14)] of $\chi$ directly implies, through Eq. (10), universal features for $\mathcal{N}_{\alpha}(E)=\mathcal{N}_{0}\left(E-\chi \Delta_{\infty}\right)$ and for the MB DOS $\rho^{(N)}(E)=$ $\rho_{0}^{(N)}\left(E-\chi \Delta_{\infty}\right)\left[1-(d / d E)\left(\chi \Delta_{\infty}\right)\right]$, both represented in 

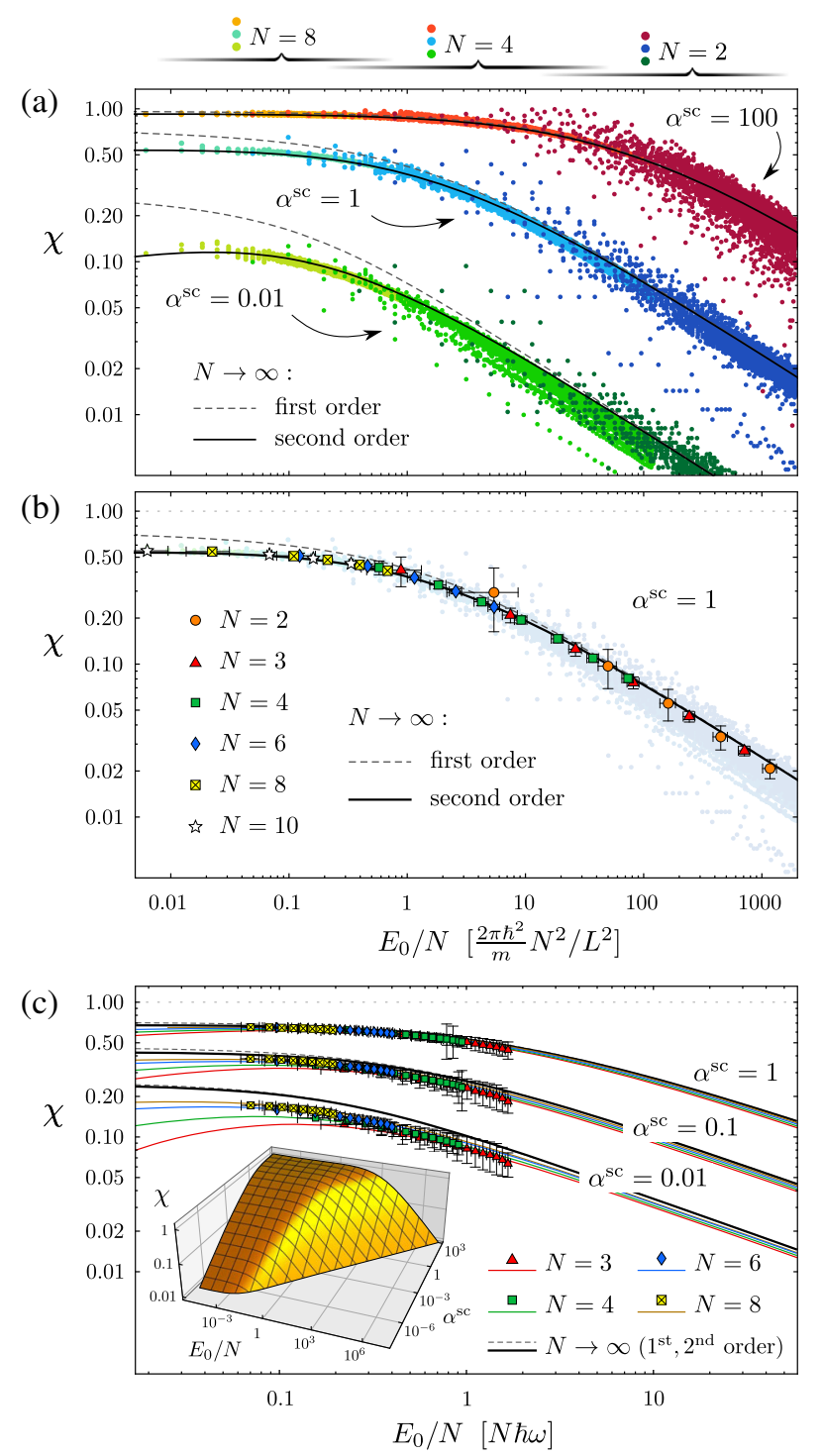

FIG. 3. Universal behavior of partial fermionization in repulsive 1D Bose gases. The ratio $\chi \in[0,1]$, Eq. (8), as a function of energy per particle $E_{0} / N$ of the noninteracting system is shown for (a),(b) the Lieb-Liniger model and (c) harmonic confinement for various couplings $\alpha$ and $N$. Numerical results for $\chi$ [dots representing individual levels in (a); symbols in (b) with bars indicating one standard deviation of local average over data chunks to obtain smooth densities] are extracted level by level from comparing the staircase functions (see, e.g., Fig. 1) of noninteracting, fully fermionized, and interacting energies $\left[\sim 10^{6}\right.$ lowest states in (a),(b) and $~ 80-200$ states in (c)]. The numerical data for different $N$ collapse to universal functions [Eq. (14)], see text for details. Inset: Universal prediction for $\chi\left(E_{0}^{\mathrm{sc}}, \alpha^{\mathrm{sc}}\right)$ for harmonic confinement.

terms of their noninteracting limits at shifted energy. (iii) Corresponding expressions for the microcanonical and canonical partition functions and thereby thermodynamic quantities follow right away. For example, Eq. (14) implies that the microcanonical temperature $T$ can be determined as well by the scaled variables [Eq. (13)].
Thus, in the thermodynamic limit $N, V_{\text {eff }} \rightarrow \infty$ with $n_{\text {eff }}$ fixed, partial fermionization $\chi\left(T, \alpha, n_{\text {eff }}\right)$ is an intensive quantity. (iv) Experimentally accessible [76] local pair correlations provide a direct probe of $\chi$ through a simple exact relation [57]. (v) Equation (5) also holds true for fermions indicating that our approach can be generalized to fermions. (vi) Another application concerns MB scattering through interacting media due to a fundamental relation between the smooth DOS and the average dwell time $[77,78]$ that is, in the single-particle case, robust against disorder implying universality [79].

To conclude we have shown that the consistent use of short-time dynamical information in the description of short-range-interacting systems enables a separation of interaction and confinement effects implying universal features of smoothed MB spectra and related thermodynamic properties. Here we have made this explicit [Eq. (12)] and have confirmed it for two models of 1D contact-interacting bosons. On top, the way universality [Eq. (14)] is derived is not restricted to 1D systems and does not depend on details of the interactions apart from being sufficiently short-ranged and excluding strongly attractive forces if supporting $\mathrm{MB}$ bound states beyond small cluster formation [80]. Hence we envisage applications and benchmarks in higher dimensions and for other types of interaction, e.g., by utilizing Beth-Uhlenbeck type formulas $[81,82]$.

We acknowledge illuminating discussions with B. Geiger, P. Schmelcher, and S. Tomsovic, and partial financial support from the Deutsche Forschungsgemeinschaft through Research Unit FOR760 and Project No. Ri689/14-1.

*quirin.hummel@ur.de

[1] M. Greiner, O. Mandel, T. Esslinger, T. W. Hänsch, and I. Bloch, Nature (London) 415, 39 (2002).

[2] F. Serwane, G. Zürn, T. Lompe, T. B. Ottenstein, A. N. Wenz, and S. Jochim, Science 332, 336 (2011).

[3] G. Zürn, F. Serwane, T. Lompe, A. N. Wenz, M. G. Ries, J. E. Bohn, and S. Jochim, Phys. Rev. Lett. 108, 075303 (2012).

[4] A. N. Wenz, G. Zürn, S. Murmann, I. Brouzos, T. Lompe, and S. Jochim, Science 342, 457 (2013).

[5] P. M. Preiss, R. Ma, M. E. Tai, A. Lukin, M. Rispoli, P. Zupancic, Y. Lahini, R. Islam, and M. Greiner, Science 347, 1229 (2015).

[6] H. Moritz, T. Stöferle, M. Köhl, and T. Esslinger, Phys. Rev. Lett. 91, 250402 (2003).

[7] T. Kinoshita, T. Wenger, and D. S. Weiss, Science 305, 1125 (2004).

[8] B. Paredes, A. Widera, V. Murg, O. Mandel, S. Folling, I. Cirac, G. V. Shlyapnikov, T. W. Hansch, and I. Bloch, Nature (London) 429, 277 (2004).

[9] A. Turpin, J. Polo, Y. V. Loiko, J. Küber, F. Schmaltz, T. K. Kalkandjiev, V. Ahufinger, G. Birkl, and J. Mompart, Opt. Express 23, 1638 (2015). 
[10] I. Bloch, J. Dalibard, and W. Zwerger, Rev. Mod. Phys. 80, 885 (2008).

[11] V. E. Korepin, N. M. Bogoliubov, and A. G. Izergin, Quantum Inverse Scattering Method and Correlation Functions, Cambridge Monographs on Mathematical Physics (Cambridge University Press, Cambridge, England, 1993).

[12] X.-W. Guan, M. T. Batchelor, and C. Lee, Rev. Mod. Phys. 85, 1633 (2013).

[13] T. Kinoshita, T. Wenger, and D. S. Weiss, Nature (London) 440, 900 (2006).

[14] F. Meinert, M. Panfil, M. J. Mark, K. Lauber, J.-S. Caux, and H.-C. Nägerl, Phys. Rev. Lett. 115, 085301 (2015).

[15] A. Polkovnikov, K. Sengupta, A. Silva, and M. Vengalattore, Rev. Mod. Phys. 83, 863 (2011).

[16] I. Brouzos and P. Schmelcher, Phys. Rev. Lett. 108, 045301 (2012).

[17] B. Wilson, A. Foerster, C. Kuhn, I. Roditi, and D. Rubeni, Phys. Lett. A 378, 1065 (2014).

[18] G. P. Brandino, J.-S. Caux, and R. M. Konik, Phys. Rev. X 5, 041043 (2015).

[19] M. A. Garcia-March, S. van Frank, M. Bonneau, J. Schmiedmayer, M. Lewenstein, and L. F. Santos, New J. Phys. 20, 113039 (2018).

[20] V. V. Kocharovsky, V. V. Kocharovsky, M. Holthaus, C. H. R. Ooi, A. Svidzinsky, W. Ketterle, and M. O. Scully, Adv. At. Mol. Opt. Phys. 53, 291 (2006).

[21] V. Guarrera, D. Muth, R. Labouvie, A. Vogler, G. Barontini, M. Fleischhauer, and H. Ott, Phys. Rev. A 86, 021601(R) (2012).

[22] J. P. Ronzheimer, M. Schreiber, S. Braun, S. S. Hodgman, S. Langer, I. P. McCulloch, F. Heidrich-Meisner, I. Bloch, and U. Schneider, Phys. Rev. Lett. 110, 205301 (2013).

[23] S. I. Mistakidis, L. Cao, and P. Schmelcher, J. Phys. B 47, 225303 (2014).

[24] G. M. Koutentakis, S. I. Mistakidis, and P. Schmelcher, Phys. Rev. A 95, 013617 (2017).

[25] H. A. Bethe, Phys. Rev. 50, 332 (1936).

[26] G. H. Hardy and S. Ramanujan, Proc. London Math. Soc. s2-17, 75 (1918).

[27] T. von Egidy, A. Behkami, and H. Schmidt, Nucl. Phys. A454, 109 (1986).

[28] T. von Egidy, H. Schmidt, and A. Behkami, Nucl. Phys. A481, 189 (1988).

[29] J. French and S. Wong, Phys. Lett. 33B, 449 (1970).

[30] O. Bohigas and J. Flores, Phys. Lett. 34B, 261 (1971).

[31] Q. Hummel, J. D. Urbina, and K. Richter, J. Phys. A 47, 015101 (2014).

[32] G. E. Andrews, Ramanujan J. 7, 385 (2003).

[33] See, e.g., Refs. [34] and [35] for recent calculations for the Sachdev-Ye-Kitaev model and quantum spin-1/2 Ising model with tilted field.

[34] A. M. García-García and J. J. M. Verbaarschot, Phys. Rev. D 96, 066012 (2017).

[35] Y. Y. Atas and E. Bogomolny, J. Phys. A 47, 335201 (2014).

[36] M. Campisi, P. Hänggi, and P. Talkner, Rev. Mod. Phys. 83, 771 (2011).

[37] J. Goold, F. Plastina, A. Gambassi, and A. Silva, in Thermodynamics in the Quantum Regime, edited by $\mathrm{F}$. Binder, L. Correa, C. Gogolin, J. Anders, and G. Adesso,
Fundamental Theories of Physics Vol. 195 (Springer, Cham, 2018), pp. 317-336.

[38] Q. Wang and H. T. Quan, Phys. Rev. E 95, 032113 (2017).

[39] E. G. Arrais, D. A. Wisniacki, L. C. Céleri, N. G. de Almeida, A. J. Roncaglia, and F. Toscano, Phys. Rev. E 98, 012106 (2018).

[40] A. Chenu, I. L. Egusquiza, J. Molina-Vilaplana, and A. del Campo, Sci. Rep. 8, 12634 (2018).

[41] Y.-Q. Li, S.-J. Gu, Z.-J. Ying, and U. Eckern, Europhys. Lett. 61, 368 (2003).

[42] A. G. Sykes, P. D. Drummond, and M. J. Davis, Phys. Rev. A 76, 063620 (2007).

[43] J. R. Armstrong, N. T. Zinner, D. V. Fedorov, and A. S. Jensen, Phys. Rev. E 85, 021117 (2012).

[44] N. J. Robinson and R. M. Konik, J. Stat. Mech. (2017) 063101.

[45] H. Weyl, Nachr. Ges. Wiss. Gött. Math.-Phys. Kl. 1911, 110 (1911).

[46] R. Balian and C. Bloch, Ann. Phys. (N.Y.) 60, 401 (1970).

[47] R. Balian and C. Bloch, Ann. Phys. (N.Y.) 64, 271 (1971).

[48] V. Ivrii, Bull. Math. Sci. 6, 379 (2016).

[49] H. D. Ursell, Math. Proc. Cambridge Philos. Soc. 23, 685 (1927).

[50] B. Kahn and G. Uhlenbeck, Physica 5, 399 (1938).

[51] P. Grüter and F. Laloë, J. Phys. I France 5, 181 (1995).

[52] F. Calogero, J. Math. Phys. (N.Y.) 12, 419 (1971).

[53] B. Sutherland, Phys. Rev. A 5, 1372 (1972).

[54] F. D. M. Haldane, Phys. Rev. Lett. 67, 937 (1991).

[55] M. V. N. Murthy and R. Shankar, Phys. Rev. Lett. 73, 3331 (1994).

[56] Z. N. C. Ha, Phys. Rev. Lett. 73, 1574 (1994).

[57] See Supplemental Material at http://link.aps.org/supplemental/ 10.1103/PhysRevLett.122.240601, Appendix A, for the derivation of Eq. (1), Appendix B, for the derivation and explicit calculation of interaction kernels, Appendix C, for the derivation and explicit calculation of Eq. (5), Appendix D, for the QCE as strong coupling expansion, Appendix E, for energy shifting, partial fermionization, and the consistency argument supporting Eq. (8), Appendix F, for large- $N$ asymptotics of the $\chi$ function, the full shift $\Delta_{\infty}^{(N, d)}$, and universality of partial fermionization, Appendix G, for the identification of the three characteristic regimes of partial fermionization and explicit approximants, Appendix $\mathrm{H}$, for the relation between local pair correlations and partial fermionization, which includes Refs. [58-61].

[58] E. B. Manoukian, J. Phys. A 22, 67 (1989).

[59] M. D. Hoffman, P. D. Javernick, A. C. Loheac, W. J. Porter, E. R. Anderson, and J. E. Drut, Phys. Rev. A 91, 033618 (2015).

[60] A. Comtet, P. Leboeuf, and S. N. Majumdar, Phys. Rev. Lett. 98, 070404 (2007).

[61] J. C. Zill, T. M. Wright, K. V. Kheruntsyan, T. Gasenzer, and M. J. Davis, SciPost Phys. 4, 011 (2018).

[62] J. Rotman, An Introduction to the Theory of Groups, Graduate Texts in Mathematics (Springer, New York, 1999).

[63] Setting $\hbar^{2} /(2 m)=1$, any coupling of nonvanishing dimension $\left[q^{\nu}\right], \nu \neq 0$, can be associated with an energy-like parameter $\alpha$, excluding only scale-invariant interactions. 
[64] Q. Hummel, Semiclassical theory of few- and manybody quantum systems with short-range interactions, Ph. D. thesis, Universität Regensburg, 2017.

[65] M. Olshanii, Phys. Rev. Lett. 81, 938 (1998).

[66] T. Bergeman, M. G. Moore, and M. Olshanii, Phys. Rev. Lett. 91, 163201 (2003).

[67] L. Tonks, Phys. Rev. 50, 955 (1936).

[68] M. Girardeau, J. Math. Phys. (N.Y.) 1, 516 (1960).

[69] T. Cheon and T. Shigehara, Phys. Rev. Lett. 82, 2536 (1999).

[70] E. H. Lieb and W. Liniger, Phys. Rev. 130, 1605 (1963).

[71] E. H. Lieb, Phys. Rev. 130, 1616 (1963).

[72] J. B. McGuire, J. Math. Phys. (N.Y.) 5, 622 (1964).

[73] Y.-Z. Jiang, Y.-Y. Chen, and X.-W. Guan, Chin. Phys. B 24, 050311 (2015).
[74] B. Geiger, Q. Hummel, J. D. Urbina, and K. Richter, Phys. Rev. A 97, 063612 (2018).

[75] B. Sutherland, J. Math. Phys. (N.Y.) 12, 246 (1971).

[76] T. Kinoshita, T. Wenger, and D. S. Weiss, Phys. Rev. Lett. 95, 190406 (2005).

[77] V. Lyuboshitz, Phys. Lett. 72B, 41 (1977).

[78] G. Iannaccone, Phys. Rev. B 51, 4727 (1995).

[79] R. Pierrat, P. Ambichl, S. Gigan, A. Haber, R. Carminati, and S. Rotter, Proc. Natl. Acad. Sci. U.S.A. 111, 17765 (2014).

[80] B. Geiger, J. D. Urbina, Q. Hummel, and K. Richter, Phys. Rev. E 96, 022204 (2017).

[81] E. Beth and G. E. Uhlenbeck, Physica 4, 915 (1937).

[82] S. Y. Larsen, M. Lassaut, and A. Amaya-Tapia, Ann. Phys. (Amsterdam) 374, 291 (2016). 